\title{
GISTs with PDGFRA exon 14 mutations represent subset of clinically favorable gastric tumors with epithelioid morphology
}

\author{
Jerzy Lasota ${ }^{1}$, Jerzy Stachura ${ }^{2}$ and Markku Miettinen ${ }^{1}$ \\ ${ }^{1}$ Department of Soft Tissue Pathology, Armed Forces Institute of Pathology, Washington, DC, USA and \\ ${ }^{2}$ Department of Pathomorhpology, Jagiellonian University, Krakow, Poland
}

\begin{abstract}
Gastrointestinal stromal tumors (GISTs) are common mesenchymal tumors of the gastrointestinal tract. Activating KIT or PDGFRA (platelet-derived growth factor receptor $\alpha$ ) mutations have been shown to be a major force in GIST pathogenesis. Recently, a previously undescribed N659K PDGFRA exon 14 mutation has been reported in GISTs. The purpose of this study was to evaluate the frequency of GISTs with PDGFRA exon 14 mutations and define the clinicopathologic profile of such tumors. In all, 200 GISTs negative for mutations in KIT exons 9, 11, 13 and 17 and PDGFRA exons 12 and 18 were evaluated for PDGFRA exon 14 mutations by PCR amplification and direct sequencing. Mutations were found in 11 of $119(9 \%)$ gastric GISTs. None of the 81 GISTs from other than gastric location had such a PDGFRA mutation. A majority of these mutations (eight cases) represented simple 2125C $>A$ or $C>G$ missense mutations, leading to substitution of the lysine for asparagine (N659K). However, in two cases, 2123A $>$ T missense mutations leading to substitution of the tyrosine for asparagine (N659Y) was found instead. Of 11 PDGFRA N659-mutant GISTs, 10 had pure epithelioid morphology. One tumor had mixed, predominantly spindle and focally epithelioid cell morphology. Frequency of PDGFRA N659-mutant GISTs among pure epithelioid GISTs was almost 19\%. Immunohistochemically, the majority (64\%) of these tumors lacked KIT expression or showed only focal scattered KIT positivity. Tumor size ranged from 2.5 to $16 \mathrm{~cm}$ (average $7.1 \mathrm{~cm}$ ). Low mitotic activity, $\leq 5$ mitoses $/ 50$ high power field was detected in six GISTs including larger, $>5 \mathrm{~cm}$ tumors. Based on mitotic activity and tumor size, six tumors were classified as probably benign with very low malignant potential. Low to moderate malignant potential and high malignant potential was suggested in three and two tumors, respectively. In four cases with moderate or high malignant potential GISTs, a long-term follow-up (average 235.5 months) showed favorable course of disease.

Laboratory Investigation (2006) 86, 94-100. doi:10.1038/labinvest.3700360; published online 24 October 2005
\end{abstract}

Keywords: PDGFRA; mutations; GIST; stomach; KIT; prognosis

Gastrointestinal stromal tumors (GISTs) are common mesenchymal tumors of the gastrointestinal (GI) tract, linked by morphologic and immunohistochemical features to the interstitial cell of Cajal, the pacemaker cells regulating GI peristalsis and autonomic nervous system. Morphologic spectrum of GISTs includes cellular, spindle cell, epithelioid or rarely pleomorphic tumors with some site-related variation. ${ }^{1}$ Immunohistochemically, a great majority

Correspondence: Dr J Lasota MD, Department of Soft Tissue Pathology, Armed Forces Institute of Pathology, 14th Street and Alaska Avenue, N.W., Washington, DC 20306-6000, USA.

E-mail: lasota@afip.osd.mil

The opinions and assertions contained herein are the expressed views of the authors and are not to be construed as official or reflecting the views of the Departments of the Army or Defense. Received 2 August 2005; revised 13 September 2005; accepted 14 September 2005; published online 24 October 2005 of GISTs show strong, diffuse KIT expression. However, in some cases KIT immunoreactivity is weak or undetectable. ${ }^{2-5}$

Activation of KIT by its ligand leads to downstream phosphorylation of substrate proteins and subsequently activates networks of signal transduction pathways that regulate cell proliferation, survival, apoptosis, motility and other important cell functions. ${ }^{6}$

Mutually exclusive gain-of-function KIT or PDGFRA (platelet-derived growth factor receptor $\alpha$ ) mutations have been documented in a majority of GISTs and are believed to be a major force in their pathogenesis. ${ }^{7,8}$ KIT and PDGFRA are members of the type III TK receptor family and display extensive structural homology. ${ }^{9}$ Pathologic activation of KIT or PDGFRA leads to enhanced proliferation and development of cancer; ${ }^{10,11}$ its inhibition is considered an important therapeutic approach in oncology. ${ }^{12}$ 
In GISTs, a great majority of KIT mutations affect the juxtamembrane (JM) domain (exon 11); however, mutations in KIT extracellular domain (exon 9) and tyrosine kinase (TK1 and TK2) domains (exons 13 and 17) have also been reported in a minority of cases. ${ }^{7,13-15}$ While KIT exon 11 mutations were found in GISTs from different locations, KIT exon 9 mutations show strong predilection to intestinal tumors. ${ }^{16,17}$

In KIT exon 9, 11, 13 and 17 wild-type GISTs, gain-of-function PDGFRA TK2 (exon 18) and JM (exon 12) mutations have been reported. ${ }^{8}$ The presence of such PDGFRA mutations has been linked to gastric location of tumor, epithelioid morphology and lack of KIT expression in some cases. $^{3-5,18}$

More recently, missense mutation in PDGFRA TK1 (exon 14) leading to N659K amino-acid substitution was reported in one gastric GIST. ${ }^{2}$ Although subsequently two more tumors with such a PDGFRA mutation were reported, ${ }^{18}$ little is known about frequency and clinicopathologic profile of GISTs carrying this type of PDGFRA mutation.

KIT/PDGFRA tyrosine kinase inhibitor imatinib mesylate (Gleevec/Glivec, formerly STI571, Novartis Pharma, Basel, Switzerland) has been successfully used to treat clinically advanced and metastatic GISTs. ${ }^{12,19,20}$ However, it has been shown that the response to such treatment can depend on KIT or PDGFRA mutation status of the tumor. ${ }^{21}$

The purpose of this study was to evaluate the frequency, morphologic features and natural history of GISTs with PDGFRA exon 14 mutations and define the clinicopathologic profile and natural history of such tumors prior to the availability of tyrosine kinase inhibitor treatment.

\section{Materials and methods}

\section{Tissue Material and Clinical Data}

In all, 200 GISTs negative for KIT exon 9, 11, 13 and 17 and PDGFRA exon 12 and 18 mutations were obtained from the files of the Armed Forces Institute of Pathology in Washington, DC and from the Department of Pathomorphology of the Jagiellonian University in Krakow in Poland.

Tumors were diagnosed using previously described morphologic and immunohistochemical criteria including evaluation of KIT (CD117), CD34, $\alpha$-smooth muscle actin (SMA), desmin (DES) and S100 protein. ${ }^{1}$ Although a great majority of cases were KIT positive, a small number of negative cases were included based on their histologic identity with positive cases. The analyzed tumors were divided into three histological types, epithelioid, spindle cell and mixed with epithelioid and spindle cell features. Based on our previously published study on gastric GISTs, pure epithelioid tumors were subclassified into following categories: sclerosing epithelioid with a syncytial pattern, epithelioid with dyscohesive pattern, hypercellular epithelioid and sarcomatous epithelioid GISTs. ${ }^{22}$

Mitoses were counted in 50 high power fields (HPF), a total area of $5 \mathrm{~mm}^{2}$. Primary GISTs were distributed into eight groups defining predicted clinical behavior based on tumor size and mitotic activity without knowledge of the outcome. The criteria used to classify tumor into clinicopathologic, prognostic groups were reported previously ${ }^{22}$ and are listed in Table 1. Clinical follow-up data were obtained and managed according to institutional review board approval. Patients treated with Gleevec/Glivec were not included in this study as case accrual antedated availability of this drug.

\section{Molecular Studies}

In all, 200 selected GISTs were evaluated for mutations affecting codon 659 in exon 14 of PDGFRA TK1. DNA was extracted from formalinfixed paraffin-embedded (FFPE) tissues and PCR amplified using primers flanking critical region of exon 14 (forward primer, 5'-GTAGCTCAGCTG GACTGATA-3'; and reverse primer, $5^{\prime}$-AATCCT CACTCCAGGTCAGT-3'). Cycling conditions and the reaction mix were the standard ones as recom-

Table 1 Tumor size and mitotic criteria used to assessed malignant potential of GISTs. Based on previously published study (Miettinen et $a l^{22}$ )

\begin{tabular}{lccl}
\hline Group & Size (in $\mathrm{cm}$ ) & Mitosis $($ per 50 HPF) & Malignant potential of gastric GISTS \\
\hline 1 & $\leq 2$ & $\leq 5$ & Benign (no metastatic risk) \\
2 & $>2$ but $\leq 5$ & $\leq 5$ & Probably benign, very low malignant potential (2-3\% metastatic risk) \\
3a & $>5$ but $\leq 10$ & $\leq 5$ & Probably benign, very low malignant potential (2-3\% metastatic risk) \\
$3 \mathrm{~b}$ & $>10$ & $\leq 5$ & Low to moderate malignant potential (11\% metastatic risk) \\
4 & $\leq 2$ & $>5$ & Uncertain or low malignant potential (too few cases to tell prognosis) \\
5 & $>2$ but $\leq 5$ & $>5$ & Low to moderate malignant potential (15\% metastatic risk) \\
$6 \mathrm{a}$ & $>5$ but $\leq 10$ & $>5$ & High malignant potential (49\% metastatic risk) \\
$6 \mathrm{~b}$ & $>10$ & High malignant potential (86\% metastatic risk)
\end{tabular}


mended by PE Applied Biosystems (Foster City, CA, USA) with annealing temperature set at $55^{\circ} \mathrm{C}$ The amplification products were purified and sequenced directly as described previously. ${ }^{23}$

\section{Results}

\section{Types, Frequency and Distribution of PDGFRA Exon 14 Mutations among GISTs}

In all, 11 tumors with a mutation affecting codon 659 were found among 200 KIT exon 9, 11, 13 and 17 and PDGFRA exon 12 and 18 mutationnegative GISTs. In eight tumors, 2125C $>$ A $(n=6)$ or $\mathrm{C}>\mathrm{G}(n=2)$ mutations leading to substitution of lysine for asparagine (N659K) were identified. In three GISTs, 2123A > T mutations leading to substitution of tyrosine for asparagine (N659Y) were identified. Nucleotide positions are based on PDGFRA mRNA sequence (locus NM_006206) available at www.ncbi.nlm.nih.gov. Examples of direct sequencing of PCR products are shown in Figure 1. All 11 PDGFRA exon 14 mutations were found among gastric GISTs. No PDGFRA exon 14 mutations were found in GISTs from other than gastric location including tumors diagnosed in esophagus $(n=2)$, small intestine $(n=68)$, large intestine $(n=9)$ mesenterium $(n=1)$ and omentum $(n=1)$. Frequency of GISTs with PDGFRA exon14 mutation among gastric GISTs negative for mutations in KIT exons 9, 11, 13 and 17 and PDGFRA exons 12 and 18 was $9 \%$; however, the frequency reached almost $19 \%$ among gastric tumors with pure epithelioid morphology $(n=53)$. Also, one of 18 GISTs with mixed spindle cell and epithelioid morphology revealed N659K mutation; however, no mutations were identified in 48 pure spindle cell gastric GISTs.

\section{Demographic and Clinicopathologic Profile of PDGFRA-Mutant Exon 14 GISTs}

There were 11 patients consisting of six males and five females (male to female ratio was 1.2:1). The patient age ranged from 48 to 90 years (median 57 years, mean 59 years).

Of 11 PDGFRA N659-mutant GISTs, 10 showed pure epithelioid morphology, and one tumor had mixed spindle cell and epithelioid cell features. In this case, spindle cell morphology dominated and epithelioid component was seen only focally. In all, 10 pure epithelioid tumors were subclassified into the following categories: sclerosing epithelioid with a syncytial pattern $(n=5)$, epithelioid with dyscohesive pattern $(n=2)$ and hypercellular epithelioid $(n=2)$. Histological examples of PDGFRA N659-mutant GISTs are shown in Figure 2.

Immunohistochemically, KIT expression was documented in six of 11 PDGFRA N659-mutant GISTs. However, in two tumors, KIT expression was limited to small cell clusters or scattered positive cells. Both diffuse cytoplasmic and perinuclear dot-like KIT expression patterns were identified. Five PDGFRA N659-mutant GISTs were KIT negative. None of the KIT-negative tumors expressed CD34. However, in one case, strong DES expression was documented without SMA being present. Two KIT-positive tumors expressed SMA. All 11 analyzed GISTs were negative for S100 protein. Complete immunohistochemical expression profile of analyzed tumors is shown in Table 2. Examples of immunohistochemical findings are shown in Figure 2.

Tumor size ranged from 2.5 to $16 \mathrm{~cm}$ with average maximum diameter of $7.1 \mathrm{~cm}$. Six PDGFRA N659mutant GISTs showed low mitotic activity $(\leq 5 /$ $50 \mathrm{HPF}$ ) including three tumors with no detectable mitotic activity (0/50 HPF). However, almost half of GISTs with PDGFRA N659-mutant GISTs (five tumors) had at least five or more mitoses per $50 \mathrm{HPF}$. The highest mitotic count recorded was 13/50 HPF.

Based on previously published criteria $^{22}$ PDGFRA N659-mutant GISTs were classified into eight prognostic groups by tumor size and mitotic activity in order to evaluate the likelihood of GIST malignant behavior. Six tumors were placed in the 2 and 3a prognostic groups and considered to be probably benign with very low malignant potential.

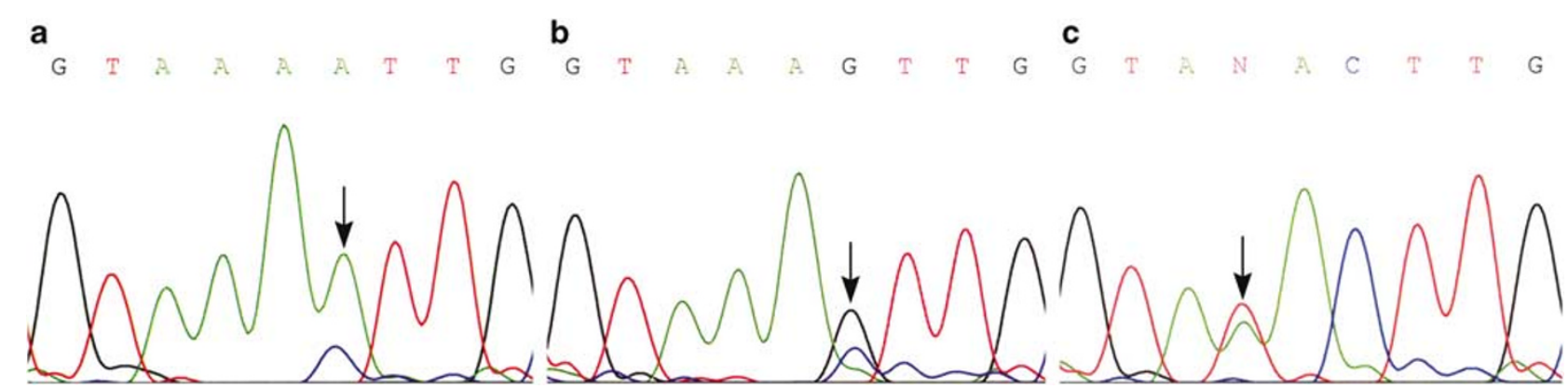

Figure 1 Examples of genomic sequences identified by direct sequencing of PCR amplification products from PDGFRA-MT exon 14 GISTs. A C $>$ A substitution (a) and a C > G substitution (b) leading to N659K mutation and an A $>$ T substitution (c) leading to N659Y mutation. 


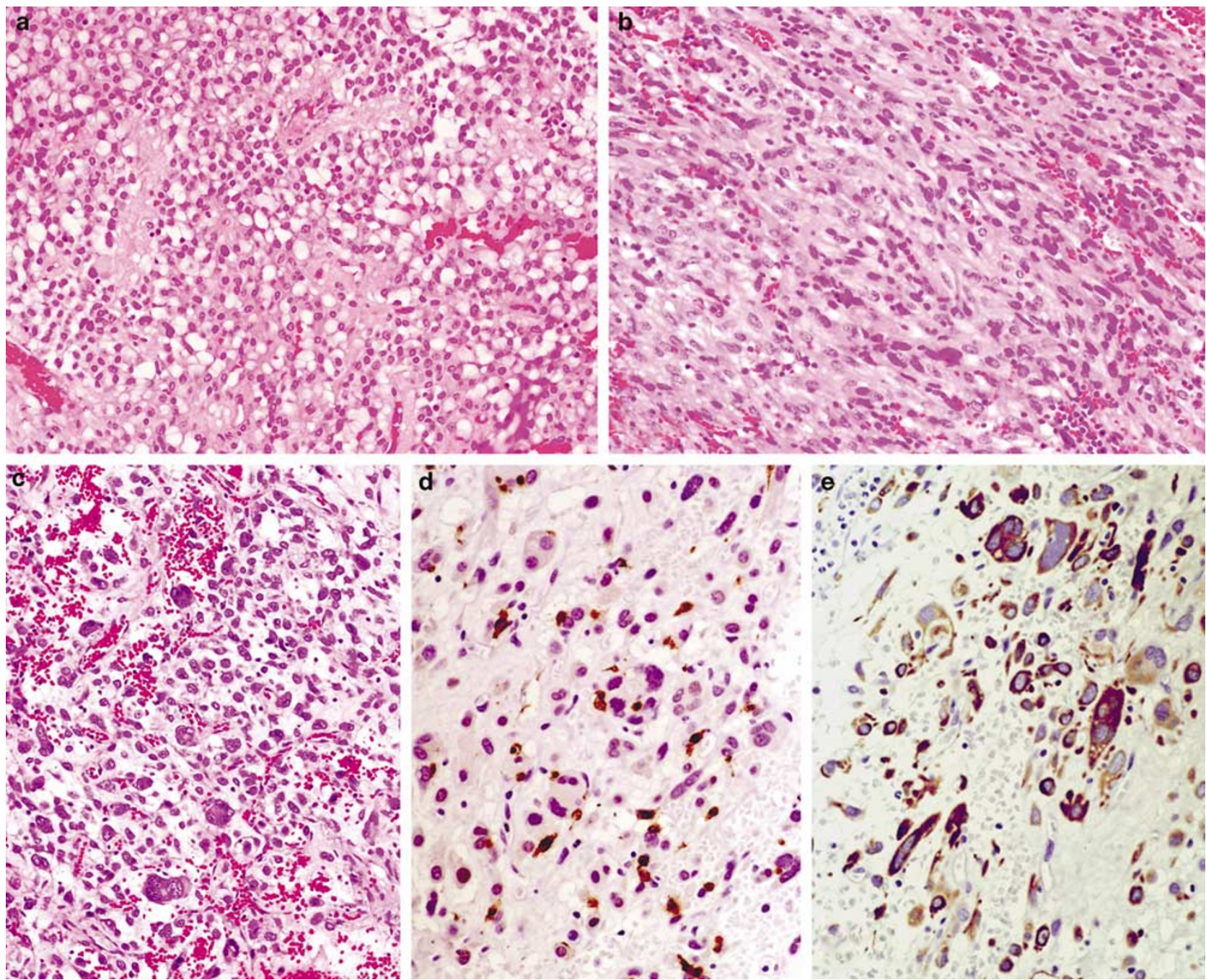

Figure 2 Examples of histological and immunohistochemical findings in PDGFRA-MT exon 14 GISTs. Sclerosing epithelioid GIST (case 11) with a syncytial pattern (a); GIST (case 8) with predominantly spindle cell morphology (b); hypercellular epithelioid GIST (case 9) with focal pleomorphism (c); lack of KIT expression and multiple KIT-positive mast cells (d); and strong diffuse DES expression (e).

Table 2 Summary of clinicopathologic data of 11 GISTs with PDGFRA-TK1 codon 659 missense point mutations

\begin{tabular}{|c|c|c|c|c|c|c|c|c|c|c|c|c|}
\hline No & Age (years)/sex & Eth & Histology & Size & Mitosis & Group & CD34 & $C D 117$ & $S M A$ & $D E S$ & PDGFRA & Follow-up \\
\hline 1 & $48 / \mathrm{F}$ & B & Ep & 2.5 & 0 & 2 & 0 & 0 & 0 & 0 & N659K & DUNK $241 \mathrm{~m}$ \\
\hline 2 & $65 / \mathrm{M}$ & Unk & Ep & 4 & 0 & 2 & 0 & $\mathrm{f}<5$ & 0 & 0 & N659K & Unk \\
\hline 3 & $49 / \mathrm{F}$ & C & $\mathrm{Ep}$ & 4 & 11 & 5 & 0 & 0 & 0 & 0 & N659K & ANED $198 \mathrm{~m}$ \\
\hline 4 & $58 / \mathrm{M}$ & C & Ep & 4.4 & 8 & 5 & 0 & 100 & 25 & 0 & N659Y & ANED $202 \mathrm{~m}$ \\
\hline 5 & $61 / \mathrm{M}$ & $\mathrm{C}$ & Ep & 4.5 & 5 & 2 & 100 & 100 & 0 & 0 & N659K & ANED $101 \mathrm{~m}$ \\
\hline 6 & $54 / \mathrm{M}$ & Unk & Ep & 5.5 & 0 & $3 a$ & 0 & 0 & 0 & 0 & N659Y & ANED $100 \mathrm{~m}$ \\
\hline 7 & $90 / \mathrm{M}$ & Unk & Ep & 6.5 & 4 & 3а & 5 & $\mathrm{f}<5$ & 0 & 0 & N659Y & DUNK $30 \mathrm{~m}$ \\
\hline 8 & $56 / \mathrm{F}$ & C & $\mathrm{Sp}+\mathrm{f} \mathrm{Ep}$ & 6.5 & 4 & 3a & 100 & 80 & 0 & 0 & N659K & ANED $27 \mathrm{~m}$ \\
\hline 9 & $58 / \mathrm{F}$ & $\mathrm{C}$ & Ep+Pleo & 9 & 7 & $6 a$ & 0 & 0 & 0 & 50 & N659K & Unk \\
\hline 10 & $50 / \mathrm{F}$ & $\mathrm{C}$ & Ep & 15 & 13 & $6 \mathrm{~b}$ & 0 & 0 & 0 & 0 & N659K & ANED $236 \mathrm{~m}$ \\
\hline 11 & $64 / \mathrm{M}$ & Unk & Ep & 16 & 2 & $3 \mathrm{~b}$ & 100 & 100 & 50 & $\mathrm{f}<5$ & N659K & ANED $206 \mathrm{~m}$ \\
\hline
\end{tabular}

Unk=unknown; $\mathrm{Ep}=$ epithelioid; $\mathrm{Sp}=$ spindle cell; Pleo=pleomorphic; $\mathrm{f}=$ focal; $\mathrm{m}=$ months; ANED=alive no evidence of disease; DUNK = died of unknown cause; $\mathrm{B}=$ Black; $\mathrm{C}=$ Caucasian

One tumor was assigned to group $3 \mathrm{~b}$ and other two to group 5 and considered to have low to moderate malignant potential. Two large $(>5 \mathrm{~cm})$ GISTs with high mitotic rate $(>5 / 50 \mathrm{HPF})$ were assigned to groups $6 \mathrm{a}$ and $6 \mathrm{~b}$ and considered to be highly malignant tumors. 


\section{Clinical Behavior of PDGFRA-Mutant Exon 14 GISTs}

Clinical follow-up data were available in nine of 11 PDGFRA N659-mutant GISTs. Four patients, one diagnosed with expectedly malignant tumor (group 6b) and three with expectedly low to moderate malignant tumors (groups $3 \mathrm{~b}$ and 5), were alive without a disease with follow-up ranging from 198 to 236 months. Two patients with low malignant potential GISTs (group 3a) were alive with follow-up 100 and 27 months, respectively. Two other patients: one with group 2 GIST and another with group 3a GIST died of unknown causes, respectively, 241 and 30 months following primary surgery. The relatively short survival of the latter patient might be related to the old patient age of $>90$ years. The clinicopathologic data from all analyzed cases are listed in Table 2.

\section{Discussion}

Gain-of-function PDGFRA exon 18 and 12 mutations were recently found in GISTs and shown to be mutually exclusive with KIT mutations. ${ }^{8}$ These mutations represent a mechanism alternative to KIT mutations for pathologic activation of tyrosine kinase receptor signaling pathways. ${ }^{8,24}$

In this study, we evaluated 200 KIT-wild-type (no mutations in exons 9, 11, 13 and 17) and PDGFRAwild-type (no mutations in exons 12 and 18) GISTs from gastric and nongastric locations for the mutations in the PDGFRA exon 14 (TK1). In all, 11 GISTs with mutation in this exon were found. All mutations involved codon 659. In eight tumors, $2125 \mathrm{C}>\mathrm{A} \quad(n=6)$ or $\mathrm{C}>\mathrm{G}(n=2)$ point mutations leading to substitution of lysine for asparagine (N659K) was identified. N659K mutation was previously reported twice,,$^{2,18}$ but no genomic sequences have been published. In three tumors, $2123 \mathrm{~A}>\mathrm{T}$ point mutation leading to previously undescribed substitution of tyrosine for asparagine (N659Y) was identified.

PDGFRA N659-mutant GISTs had a specific clinicopathologic profile showing predilection to gastric over intestinal location and a strong association with epithelioid tumor cell morphology. Associations between the presence of PDGFRA exon 18 or 12 mutations and clinicopathologic features of GISTs have been recently shown. ${ }^{3}$ Tumors with these mutations were found to be associated with gastric location and epithelioid morphology. ${ }^{3-5,18,25}$ Considering the previous and current study, it appears that the presence of any type of PDGFRA mutation is associated with gastric location and predominantly epithelioid cytologic features. However, a few exceptions have been reported including occasional finding of PDGFRA-mutant GISTs in intestines, mesentery and omentum..$^{2,3,5,18}$

Although this study was not designed to evaluate the overall frequency of the N659-mutant, we have estimated its frequency in all GISTs as no greater than $1 \%$. This estimate was based on the series of almost 700 gastric tumors evaluated for KIT exon 9, 11, 13 and 17 and PDGFRA exon 12, 14 and 18 mutations and two assumptions: (1) that nongastric GIST are negative for PDGFRA exon 14 mutation and (2) that the ratio between gastric and nongastric tumors in the general population is $1.44: 1 .^{26}$ Recently, $0.3 \%$ frequency of N659K mutation was reported in a series of 1105 GISTs with unspecified histologic type and location. ${ }^{18}$ Association of N659mutant with gastric tumor location, epithelioid morphology and benign course of disease, as shown in this study, will influence overall frequency of this mutation in GIST series depending on their composition. Thus, series with substantial number of malignant tumors from clinical trials, or series with over-representation of nongastric GISTs might show a lower frequency than series on GISTs from general population. Accurate determination of the frequency of any particular mutation in GISTs requires a large population-based study.

Gastric location and epithelioid cell morphology was also found to be typical for GISTs developing in children and young adults. ${ }^{27}$ In our series of 44 such GISTs, 32 tumors (73\%) had epithelioid cell features. Of these tumors, 10 were included in the current study and screened for PDGFRA exon 14 mutations. However, no mutations were found. This supports a notion that a different molecular mechanisms lead to the development of morphologically identical tumors in children and adults.

Based on Western blot studies, PDGFRA expression was reported in PDGFRA-mutant GISTs. ${ }^{8,24}$ However, PDGFRA expression was also documented in the number of KIT-wild-type and KIT-mutant GISTs ${ }^{8,24}$ with the tendency to be stronger in KITwild-type GISTs. ${ }^{24}$ An immunohistochemical detection of PDGFRA expression in FFPE tissues appears not reliable in our experience. Also, others have reported poor results in immunohistochemical evaluation of PDGFRA expression in FFPE GISTs and concluded that a variety of commercially available antibodies failed to produce reproducible results. ${ }^{2}$ However, recently, detection of PDGFRA expression in FFPE tissues using polyclonal antibody provided by Santa-Cruz Biotechnology Inc. (Santa Cruz, CA, USA) has been reported. ${ }^{28,29}$ Moreover perinuclear dot-like expression pattern was linked to the tumors with mutations in PDGFRA exon 12 or $18 .^{28}$ More studies are required to validate the recently published observations on immunohistochemical detection of PDGFRA in FFPE GISTs.

In this study, immunohistochemical KIT expression was evaluated in all analyzed tumors. In four cases, diffuse KIT expression was found, whereas seven of $11(64 \%)$ PDGFRA N659-mutant GISTs showed lack of KIT expression or presence of only scattered KIT-positive cells. In contrast, lack of KIT expression or scattered KIT-positive cells were found only in $22.3 \%$ of GISTs with PDGFRA exon 
18 and 12 mutations. ${ }^{3}$ Although the number of analyzed GISTs with PDGFRA exon 14 mutations is relatively small, the difference in KIT expression between these to cohorts is striking. Hirota et $a l^{24}$ suggested that constitutively activated PDGFRA mutants can bind and activate KIT-wild type in 'in vitro' transfection systems and in 'in vivo' tumor tissue as well. This study and previously published data indicate that such phenomenon does not occur in all PDGFRA-mutant GISTs. ${ }^{2}$ Further studies based on fresh/frozen tissues are necessary to understand differences between different PDGFRA-mutant GISTs.

Based on immunohistochemical studies, at least two different patterns of KIT expression: diffuse cytoplasmic and perinuclear dot-like expressions have been reported in gastric GISTs. ${ }^{27} \mathrm{~A}$ recent study showed strong association between perinuclear dotlike KIT expression pattern and presence of KIT mutation, and suggested that such a pattern is a diagnostic feature of KIT-mutant GISTs. ${ }^{28}$ PDGFRA N659-mutant GISTs, analyzed in this study, showed either diffuse or focal perinuclear dot-like KIT expression pattern. Based on our previous experience $^{1,22}$ and the current study, perinuclear dot-like KIT expression pattern is not necessarily associated with the presence of KIT mutation.

In this study, six of the analyzed PDGFRA N659mutant GISTs have been considered to be benign or have a very low malignant potential. Three GISTs were assigned either to prognostic group $3 b(n=1)$ or $5(n=2)$ of low to moderate malignant potential, and two tumors were classified as highly malignant, characterized by $>5 \mathrm{~cm}$ and $>5 / 50 \mathrm{HPF}$ mitotic rate. However, none of the four GISTs assigned to groups $3 \mathrm{~b}, 5$ and $6 \mathrm{~b}$, with long clinical follow-up (198-236 months), revealed signs of progressive disease. This observation may suggest that the presence of PDGFRA exon 14 mutations is associated with a more likely benign clinical outcome than expected based on comparison with other gastric GISTs with similar parameters. ${ }^{22}$ Study based on larger number of cases with long-term follow-up is required to confirm this hypothesis.

In our previous study, gastric GISTs with PDGFRA exon 18, D842V mutation, had a lower mitotic rate, smaller size and favorable outcome compared to the tumors with deletions. ${ }^{3}$ Also, gastric GISTs with point mutations in KIT-JM were shown to have substantially lower rate of progressive disease than the ones with the deletions. ${ }^{22}$ All these data suggest that type of PDGFRA or KIT mutation correlates with clinical outcome in gastric tumors. However, further studies are necessary to determine whether differences in clinical outcome are related to different types of activation of PDGFRA and KIT downstream signaling pathways by particular mutations, or are related to other factors.

Although PDGFRA N659-mutant GISTs follow benign course of disease, imatinib sensitivity of these tumors is an important clinical issue. Recent 'in vitro' studies showed that PDGFRA isoform with substitution N659K showed decreased phosphorylation in the presence of imatinib. ${ }^{18}$ A degree of imatinib sensitivity was similar to the one observed in the experiment with homologous KIT TK1 isoform. ${ }^{18,21}$ This observation indicates that patients with metastatic or unresectable PDGFRA N659mutant GISTs are prime candidates for imatinib (Gleevec/Glivec) treatment.

In summary, we have shown that PDGFRA N659mutant GISTs represent a small subset of gastric tumors with predominantly epithelioid cell morphology. Although almost half of these tumors were classified as expectedly malignant, long-term follow-up data suggested a benign course of disease. Therefore, detection of PDGFRA exon 14 mutations may represent an additional marker that identifies gastric GISTs with a high probability of benign behavior. However, additional studies based on larger number of cases are necessary to understand the differences between PDGFRA N659-mutant GISTs and other gastric GISTs.

\section{Acknowledgements}

This study was partially supported by the Grant from the Polish Committee for the Scientific Research (3PO5C05925) and by the American Registry of Pathology.

\section{References}

1 Miettinen M, Lasota J. Gastrointestinal stromal tumors (GISTs): definition, occurrence, pathology, differential diagnosis and molecular genetics. Pol J Pathol 2003; 54:3-24.

2 Medeiros F, Corless CL, Duensing A, et al. KIT-negative gastrointestinal stromal tumors. Proof of concept and therapeutic implications. Am J Surg Pathol 2004;28: 889-894.

3 Lasota J, Dansonka-Mieszkowska A, Sobin LH, et al. A great majority of GISTs with PDGFRA mutations represent gastric tumors of low or no malignant potential. Lab Invest 2004;84:874-883.

4 Debiec-Rychter M, Wasag B, Stul M, et al. Gastrointestinal stromal tumours (GISTs) negative for KIT (CD117 antigen) immunoreactivity. J Pathol 2004;202: 430-438.

5 Wardelmann E, Hrychyk A, Merkelbach-Bruse S, et al. Association of platelet-derived growth factor receptor $\alpha$ mutations with gastric primary site and epithelioid or mixed cell morphology in gastrointestinal stromal tumors. J Mol Diagn 2004;6:197-204.

6 Rommstrand L. Signal transduction via the stem cell factor receptor/c-KIT. Cell Mol Life Sci 2004;61: 2535-2548.

7 Hirota S, Isozaki K, Moriyama Y, et al. Gain-of-function mutations of c-kit in human gastrointestinal stromal tumors. Science 1998;279:577-580.

8 Heinrich MC, Corless CL, Duensing A, et al. PDGFRA activating mutations in gastrointestinal stromal tumors. Science 2003;299:708-710. 
9 Pawson T. Regulation and targets of receptor tyrosine kinases. Eur J Cancer 2002;38(Suppl 5):S3-S10.

10 Kitamura Y, Hirotab S. Kit as a human oncogenic tyrosine kinase. Cell Mol Life Sci 2004;61:2924-2931.

11 Corless CL, Fletcher JA, Heinrich MC. Biology of gastrointestinal stromal tumors. J Clin Oncol 2004;22: 3813-3825.

12 Demetri GD, von Mehren M, Blanke CD, et al. Efficacy and safety of imatinib mesylate in advanced gastrointestinal stromal tumors. N Engl J Med 2002;347: $472-480$.

13 Lasota J, Jasinski M, Sarlomo-Rikala M, et al. Mutations in exon 11 of c-kit occur preferentially in malignant versus benign gastrointestinal stromal tumors and do not occur in leiomyomas or leiomyosarcomas. Am J Pathol 1999;154:53-60.

14 Lux ML, Rubin BP, Biase TL, et al. KIT extracellular and kinase domain mutations in gastrointestinal stromal tumors. Am J Pathol 2000;156:791-795.

15 Rubin BP, Singer S, Tsao C, et al. KIT activation is a ubiquitous feature of gastrointestinal stromal tumors. Cancer Res 2001;61:8118-8121.

16 Lasota J, Kopczynski J, Sarlomo-Rikala M, et al. KIT 1530ins6 mutation defines subset of predominantly malignant gastrointestinal stromal tumors (GIST) of intestinal origin. Hum Pathol 2003;34:1306-1312.

17 Antonescu CR, Sommer G, Sarran L, et al. Association of KIT exon 9 mutations with non-gastric primary site and aggressive behavior: KIT mutation analysis and clinical correlates of 120 gastrointestinal stromal tumors. Clin Cancer Res 2003;15:3329-3337.

18 Corless CL, Schroeder A, Griffith D, et al. PDGFRA mutations in gastrointestinal stromal tumors: frequency, spectrum and in vitro sensitivity to imatinib. J Clin Oncol 2005;23:1-8.

19 Blay JY, Bonvalot S, Casali P, et al. Consensus meeting for the management of gastrointestinal stromal tumors. Report of the GIST Consensus Conference of 20-21 March 2004, under the auspices of ESMO. Ann Oncol 2005;16:566-578.

20 Blanke CD, Corless CL. State-of-art therapy for gastrointestinal stromal tumors. Cancer Invest 2005;23: 274-280.
21 Heinrich MC, Corless CL, Demetri GD, et al. Kinase mutations and imatinib response in patients with metastatic gastrointestinal stromal tumor. J Clin Oncol 2003;23:4342-4349.

22 Miettinen M, Sobin LH, Lasota J. Gastrointestinal stromal tumors of the stomach. A clinicopathologic, immunohistochemical, and molecular genetic study of 1765 cases with long-term follow-up. Am J Surg Pathol 2005;29:52-68.

23 Lasota J, Wozniak A, Sarlomo-Rikala M, et al. Mutations in exons 9 and 13 of KIT gene are rare events of gastrointestinal stromal tumors. A study of 200 cases. Am J Pathol 2000;157:1091-1095.

24 Hirota S, Ohashi A, Nishida T, et al. Gain-of-function mutations of platelet-derived growth factor receptor $\alpha$ gene in gastrointestinal stromal tumors. Gastroenterology 2003;125:660-667.

25 Wasag B, Debiec-Rychter M, Pauwels P, et al. Differential expression of KIT/PDGFRA mutant isoforms in epithelioid and mixed variants of gastrointestinal stromal tumors depends predominantly on the tumor site. Mod Pathol 2004;17:889-894.

26 Nilsson B, Bumming P, Meis-Kindblom JM, et al. Gastrointestinal stromal tumors: the incidence, prevalence, clinical course, and prognostication in the preimatinib mesylate era. Cancer 2005;103: 821-829.

27 Miettinen M, Lasota J, Sobin LH. Gastrointestinal stromal tumors of the stomach in children and young adults: a clinicopathologic, immunohistochemical, and molecular genetic study of 44 cases with longterm follow-up and review of the literature. Am J Surg Pathol 2005;29:1373-1381.

28 Pauls K, Merkelbach-Bruse S, Thal D et al. PDGFR $\alpha-$ and c-kit-mutated gastrointestinal stromal tumours (GISTs) are characterized by distinctive histological and immunohistochemical features. Histopathology 2005;46:166-175.

29 Rossi G, Valli R, Bertolini F, et al. PDGFRA expression in differential diagnosis between KIT-negative gastrointestinal stromal tumours and other primary soft-tissue tumours of the gastrointestinal tract. Histopathology 2005;46:522-531. 